\title{
AN ALGORITHMICALLY UNSOLVABLE PROBLEM IN ANALYSIS
}

\author{
A. LENARD AND J. STILLWELL
}

\begin{abstract}
The decision problem of distinguishing between the cases when the Laplace-Beltrami operator on the covering space of a compact manifold has 0 in its spectrum or is bounded away from 0 is algorithmically unsolvable in any class of manifolds that includes all 4-dimensional ones. The proof depends on a result of Brooks connecting the spectrum with the amenability of the fundamental group.
\end{abstract}

Algorithmically unsolvable problems abound in logic, algebra, combinatorial topology, and diophantine equations (for a review and references see, for instance, [1]). In this note we point out an algorithmically unsolvable decision problem whose natural context is analysis on Riemannian manifolds.

Let $M$ be a compact differentiable manifold, $\pi_{1}(M)$ its fundamental group, $\tilde{M}$ its universal cover. If $g$ is a Riemannian metric on $M$ (and so also on $\tilde{M}$ ), let $\lambda_{0}(\tilde{M}, g$ ) denote the infimum of the spectrum of the Laplace-Beltrami operator $-\Delta$ acting in $L^{2}(\tilde{M}, g)$. Whether $\lambda_{0}(\tilde{M}, g)=0$ or $\lambda_{0}(\tilde{M}, g)>0$ holds is independent of $g$ and is, therefore, a diffeomorphism invariant of $M$. The following question then arises: Is there an algorithm for deciding which of the two cases holds? To make this question meaningful one considers a recursively enumerable class $C$ of compact differentiable manifolds, i.e. one that can be put into one-to-one correspondence with a recursively enumerable set of positive integers which serve as code names for the manifolds (for this notion see [2, §II.1]). An algorithm is then understood to be a Turing machine which, when presented with the code number of any $M$ in $C$, computes in a finite number of steps which of the above alternatives holds.

THEOREM. If $C$ is such that for every finitely presented group $G$ there is an $M$ in $C$ with $\pi_{1}(M)=G$, then there is no algorithm for the above decision problem.

The proof depends on a recent theorem of Brooks [3] which establishes a connection between the condition $\lambda_{0}(\tilde{M})=0$ and $\pi_{1}(M)$, namely, the condition holds if and only if $\pi_{1}(M)$ is an amenable group. Thus, to prove our theorem, it is only necessary to show that amenability is an algorithmically undecidable property in the class of finitely presented groups. Amenability may be defined in various ways (see [4]), but from our point of view the essential fact is that amenability is a so-called Markov property. A property $\mathbf{P}$ of finitely presented groups is called a Markov property whenever (A) there is at least one finitely presented group $G_{1}$ with the

Received by the editors June 9, 1982.

1980 Mathematics Subject Classification. Primary 03D35; Secondary 31C12, 58C40.

Key words and phrases. Unsolvability, Laplacian on manifolds, spectrum on Laplacian, amenability, Markov property. 
property $\mathrm{P}$, and (B) there is at least one finitely presented group $G_{2}$ such that $G_{2}$ is not a subgroup of any finitely presented group $G$ with property $\mathrm{P}$. In the case where $\mathrm{P}$ is amenability, one may take for $G_{1}$ the free abelian group with two generators (i.e. the group presented by the sole relation $a b a^{-1} b^{-1}=1$ ), and for $G_{2}$ the free nonabelian group with two generators. Then (A) and (B) above follow from Theorems 1.2.1, 1.2.5 and Example 1.2.3 of [4]. The following general theorem is known: There is no algorithm for deciding whether an arbitrary finitely presented group has or has not a given Markov property. (For proof see [2, §IV.4].) Thus, in particular, amenability is an algorithmically undecidable property in the class of finitely presented groups, and so our theorem follows.

We remark that the hypothesis that $C$ be a recursively enumerable class is no great restriction because it has been shown [5] that up to homeomorphism manifolds may be replaced by algebraic manifolds, i.e. loci in Euclidean spaces of algebraic equations with rational coefficients.

We also note that every finitely presented group is the fundamental group of some compact 4-dimensional manifold (see [6, p. 143]). Hence our decision problem is already algorithmically unsolvable when restricted to 4-dimensional manifolds. Whether this is still true when 4 is replaced by 3 remains an open problem.

\section{REFERENCES}

1. J. Stillwell, The word problem and the isomorphism problem for groups, Bull. Amer. Math. Soc. 6 (1982), 33-56.

2. R. C. Lyndon and P. E. Schupp, Combinatorial group theory, Ergeb. Math. Grenzgeb., No. 89, Springer-Verlag, Berlin, 1977.

3. R. Brooks, Amenability and the spectrum of the Laplacian, Bull. Amer. Math. Soc. 6 (1982), 87-79.

4. F. P. Greenleaf, Invariant means on topological groups, Van Nostrand Math. Studies No. 16, Van Nostrand, Princeton, N. J., 1969.

5. W. W. Boone, W. Haken and V. Poenaru, On recursively unsolvable problems in topology and their classification, Contributions to Mathematical Logic (H. A. Schmidt, K. Schutte, H. J. Thiele, editors), North-Holland, Amsterdam, 1968.

6. W. S. Massey, Algebraic topology: An introduction, Harcourt, Brace \& World, New York, 1967.

DEPARTMENT OF MATHEMATICS, INDIANA UNIVERSiTy, BloOMINGTON, INDIANA 47405

Department of Mathematics, Monash University, Clayton, Victoria, Australia 3168 